\title{
Hydrogen-rich saline promotes motor functional recovery following peripheral nerve autografting in rats
}

\author{
YONG-GUANG ZHANG ${ }^{1 *}$, QING-SONG SHENG ${ }^{2 *}$, ZHI-JUN WANG ${ }^{3 *}$, \\ $\mathrm{LI} \mathrm{LV}^{1}$, WEI ZHAO ${ }^{4}$, JIAN-MEI CHEN ${ }^{1}$ and HAO XU ${ }^{1}$ \\ Departments of ${ }^{1}$ Orthopedics and ${ }^{2}$ Obstetrics and Gynecology, Fuzhou General Hospital of Nanjing Military \\ Command/Dongfang Hospital of Xiamen University, Fuzhou, Fujian 350025; ${ }^{3}$ Department of Radiology, \\ General Hospital of Ningxia Medical University; ${ }^{4}$ Key Laboratory of Reproduction and Heredity \\ of Ningxia Hui Autonomous Region, Department of Biochemistry and Molecular Biology, Ningxia \\ Medical University, Yinchuan, Ningxia Hui Autonomous Region 750004, P.R. China
}

Received August 19, 2014; Accepted May 1, 2015

DOI: 10.3892/etm.2015.2518

\begin{abstract}
Despite the application of nerve grafts and considerable microsurgical innovations, the functional recovery across a long peripheral nerve gap is generally partial and unsatisfactory. Thus, additional strategies are required to improve nerve regeneration across long nerve gaps. Hydrogen possesses antioxidant and anti-apoptotic properties, which could be neuroprotective in the treatment of peripheral nerve injury; however, such a possibility has not been experimentally tested in vivo. The aim of the present study was to investigate the effectiveness of hydrogen-rich saline in promoting nerve regeneration after $10-\mathrm{mm}$ sciatic nerve autografting in rats. The rats were randomly divided into two groups and intraperitoneally administered a daily regimen of $5 \mathrm{ml} / \mathrm{kg}$ hydrogen-rich or normal saline. Axonal regeneration and functional recovery were assessed through a combination of behavioral analyses, electrophysiological evaluations, Fluoro-Gold ${ }^{\text {TM }}$ retrograde tracings and histomorphological observations. The data showed that rats receiving hydrogen-rich saline achieved better axonal regeneration and functional recovery than those receiving normal saline. These findings indicated that hydrogen-rich saline promotes nerve regeneration across long gaps, suggesting that hydrogen-rich saline could be used as a neuroprotective agent for peripheral nerve injury therapy.
\end{abstract}

Correspondence to: Dr Hao Xu or Dr Jian-Mei Chen, Department of Orthopedics, Fuzhou General Hospital of Nanjing Military Command/Dongfang Hospital of Xiamen University, 156 West Ring Road, Fuzhou, Fujian 350025, P.R. China

E-mail: xuhao_fgh@163.com

E-mail: orthospine@163.com

*Contributed equally

Key words: hydrogen, sciatic nerve, functional recovery, nerve gap

\section{Introduction}

Peripheral nerve injuries are extremely common in clinical practice. Despite a capacity for spontaneous axonal regeneration, the regenerating axons struggle to overcome a long gap into the distal stump to restore function without surgical intervention. Consequently, individuals with long nerve gaps suffer lifelong disabilities. Nerve auto graft implantation is presently the most common repair strategy for long gaps and remains the standard treatment in clinical settings (1). In addition, a number of tissue-engineering nerve grafts (TENGs) have been approved for clinical use in peripheral nerve repair (2); however, the outcomes of nerve regeneration and functional recovery using either nerve autografts or TENGs are generally partial and unsatisfactory (3-5). Additional strategies are therefore necessary to favor nerve regeneration and functional recovery across long gaps.

Pharmacotherapy is an important adjunctive treatment for peripheral nerve injury. Several neuroprotective drugs have been developed, which promote nerve regeneration experimentally and clinically. Notably, numerous drugs exert neuroprotective effects through antioxidant or anti-apoptotic mechanisms (6); therefore, substances that possess antioxidant and anti-apoptotic properties have potential for therapeutic applications following peripheral nerve injury.

In recent years, medical applications of hydrogen have attracted considerable attention. Hydrogen efficiently reduces levels of reactive oxygen (ROS) species in vitro and exerts therapeutic antioxidant activity in vivo (7-9). Additional studies have indicated that hydrogen exerts neuroprotective effects through the suppression of oxidative stress and the reduction of neuronal apoptosis in the central nervous system (10-12). To the best of our knowledge, the application of hydrogen for the treatment of peripheral nerve injury has rarely been reported. The aim of the present study, therefore, was to investigate the effectiveness of hydrogen-rich saline on motor function recovery following the reconstruction of a $10-\mathrm{mm}$ sciatic nerve gap, repaired with a nerve autograft. A combination of behavioral analyses, electrophysiological evaluations, Fluoro-Gold ${ }^{\mathrm{TM}}(\mathrm{FG})$ retrograde tracing and 
histomorphological observations of the regenerated nerves and gastrocnemius muscles were performed to assess the axonal regeneration and functional recovery.

\section{Materials and methods}

Preparation of hydrogen-rich saline. To prepare the hydrogen-rich saline, purified hydrogen was dissolved into normal saline for $4 \mathrm{~h}$ under high pressure $(0.4 \mathrm{MPa})$, as previously described (13). Hydrogen was produced using a hydrogen-generating apparatus (Shandong Saikesaisi Hydrogen Energy Co., Ltd., Shandong, China). The hydrogen-saturated saline was subsequently sterilized through exposure to $20 \mathrm{kGy}{ }^{60} \mathrm{Co}$ radiation and stored under atmospheric pressure at $4^{\circ} \mathrm{C}$.

Animal groups and surgical procedures. A total of 60 female Sprague Dawley rats (weighing 200-220 g), obtained from the Experimental Animal Center of the Fourth Military Medical University (FMMU; Xi'an, China), were anesthetized through an intraperitoneal injection of $2 \%$ sodium pentobarbital solution $(40 \mathrm{mg} / \mathrm{kg}$ body weight). Under aseptic conditions, the right sciatic nerve was exposed using a gluteal muscle-splitting incision. A $10-\mathrm{mm}$ segment of the sciatic nerve was excised and reversed under a surgical microscope to bridge the nerve gap. The wound was closed with 6-0 stitches, and the rats were transferred back to the animal room under standard housing conditions. All rats were subsequently administered an intraperitoneal injection of $5 \mathrm{ml} / \mathrm{kg}$ hydrogen saline (experimental group) or an equal volume of normal saline (control group) daily. All experimental procedures were approved by the Ethics Committee of the FMMU.

Behavioral analysis. Motor function recovery of the sciatic nerve was evaluated using the sciatic function index (SFI) on the rats at 4,8 and 12 weeks after surgery according to the method previously described $(14,15)$. Briefly, the rats $(n=10$ in each group at each time-point) were trained preoperatively to walk down a narrow wooden track ( $80 \mathrm{~cm}$ long and $7 \mathrm{~cm}$ wide) into a darkened goal box. Following surgery, all rats were directed to walk down the wooden track again, and the hind paws of each animal were painted with red dye. Pawprints were recorded on blank paper. The distance from the heel to the top of the third toe (PL), the distance between the second and the fourth toe (ITS) and the distance between the first and the fifth

$$
\mathrm{SFI}=-38.3 \times \frac{\mathrm{EPL}-\mathrm{NPL}}{\mathrm{NPL}}+109.5 \times \frac{\mathrm{ETS}-\mathrm{NTS}}{\mathrm{NTS}}+13.3 \times \frac{\text { EITS }-\mathrm{NITS}}{\mathrm{NITS}}-8.8
$$

toe (TS) were measured. E represents the experimental foot, and $\mathrm{N}$ represents the contralateral normal foot. The SFI was measured according to the following equation:

Electrophysiological evaluation. Subsequent to behavioral analyses, the rats $(n=6)$ were anesthetized and subjected to electrophysiological tests (16). The sciatic nerve was exposed under a surgical microscope and insulated from the surrounding muscles with a rubber dam. To record the compound muscle action potentials (CMAPs), the bipolar stimulating electrode (Zhongshi Dichuang Science and Technology Development
Co., Ltd., Beijing, China) was placed at the proximal portion of the sciatic nerve, and the recording electrode was placed in the gastrocnemius belly. For quantitative analysis, the CMAP peak amplitude, the CMAP latency of onset and the nerve conduction velocity $(\mathrm{NCV})$ were calculated.

Histomorphological analysis. At 4, 8 and 12 weeks after surgery, the regenerated nerve of the affected leg was harvested under anesthesia. The nerve samples $(n=6)$ were fixed with $3 \%$ glutaraldehyde, postfixed in $1 \%$ osmium tetroxide in $0.1 \mathrm{M}$ sodium cacodylate buffer and subsequently dehydrated and embedded in resin according to a standard protocol. From the distal portion of the sample, $1-\mu \mathrm{m}$ transverse semi-thin sections were cut, stained with a $1 \%$ toluidine blue/ $1 \%$ borax solution and analyzed with a light microscope. In addition, 50-nm ultrathin sections, stained with uranyl acetate/lead citrate, were prepared and analyzed with a transmission electron microscope. The following parameters were measured: Total number of myelinated axons, mean diameter of nerve fibers and degree of myelination (G-ratio).

The gastrocnemius muscles of the operated and contralateral control sides $(n=6)$ were harvested at 12 weeks after surgery, fixed in buffered $4 \%$ paraformaldehyde and subjected to hematoxylin and eosin staining. Images were subsequently captured under a light microscope (AH3; Olympus Corp., Tokyo, Japan). For each sample, the cross-sectional area of the muscle fibers was measured with images captured of 4 random fields and analyzed with the Leica software package (Leica Microsystems GmbH, Wetzlar, Germany) (17). The average percentage of muscle fiber area $\left(\mathrm{P}_{m}\right)$ was determined according to the following equation: $\mathrm{P}_{m}=\mathrm{A}_{m} / \mathrm{A}_{t} \times 100 \%$, where $\mathrm{A}_{m}$ represents the muscle fiber area in each field (magnification, x200), and $\mathrm{A}_{t}$ represents the total area in the same field.

Retrograde tracing with FG. Retrograde tracing was performed as previously described (18). Briefly, the sciatic nerve of the operated leg $(n=4)$ was exposed at 4,8 and 12 weeks after surgery, and $5 \mu \mathrm{l} 3 \%$ FG dye (Biotium, Hayward, CA, USA) solution was intraneurally injected into the nerve trunk at a site $2 \mathrm{~mm}$ proximal to the bifurcation. The incision was sutured and all rats were returned to their cages. After 5 days, the rats were anesthetized and perfused intracardially with paraformaldehyde. The L4, L5 and L6 segments of the lumbar spinal cord, together with the dorsal root ganglia (DRG), were harvested. The samples were subsequently prepared according to a standard protocol and sectioned on a cryostat. Transverse sections of $25-\mu \mathrm{m}$ thickness were obtained from the spinal cord samples, and $16-\mu \mathrm{m}$ longitudinal sections of the DRG were mounted on glass slides and analyzed with a fluorescence microscope (BX-60; Olympus Corp.). The numbers of back-labeled motoneurons and sensory neurons were counted.

Data analysis. Data are expressed as the mean \pm standard error of the mean and analyzed using one-way analysis of variance with the SPSS 13.0 software package (SPSS Inc., Chicago, IL, USA). Statistically significant overall differences among the groups were further analyzed through pair-wise comparisons with Tukey's post hoc test. The differences were considered statistically significant at $\mathrm{P}<0.05$. 

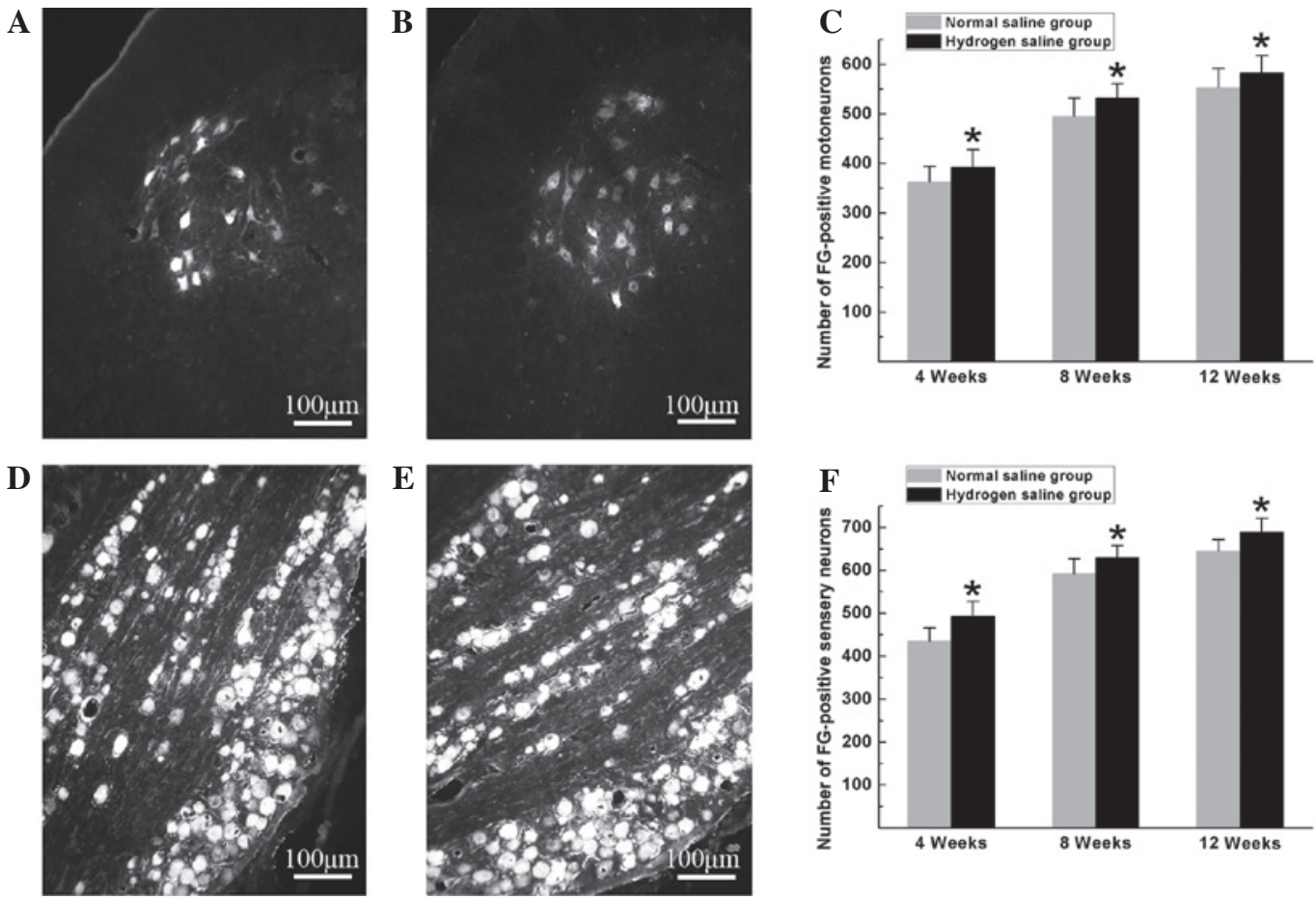

Figure 1. (A and B) Representative images of FG-positive motoneurons in the (A) normal saline and (B) hydrogen saline groups at 12 weeks after surgery. (C) The total number of FG-positive motoneurons, which regenerated into the distal nerve stump, was plotted as a function of time after surgery, (D and E) Representative images of FG-positive sensory neurons in the (D) normal saline and (E) hydrogen saline groups at 12 weeks after surgery. (F) The total number of FG-positive sensory neurons, which regenerated into the distal nerve stump, was plotted as a function of time after surgery. All data are expressed as the mean \pm standard error of the mean. ${ }^{*} \mathrm{P}<0.05$ for the comparison with the normal saline group. FG, Fluoro-Gold ${ }^{\mathrm{TM}}$.

\section{Results}

Hydrogen-rich saline enhances axonal regeneration. Hydrogen-rich saline exerted beneficial effects on neuron survival and axonal outgrowth. At the pre-indicated time-points after surgery, FG-positive motoneurons and sensory neurons were counted to identify the number of surviving neurons regenerated through the graft into the distal stump. As shown in Fig. 1, the numbers of FG-positive motoneurons and sensory neurons in the hydrogen saline group were significantly higher than those in the normal saline group $(\mathrm{P}<0.05)$, indicating that more neurons were maintained under viable conditions and more nerve fibers were generated and successfully regenerated through the graft into the distal stump in the hydrogen saline group.

Histomorphological observation of the regenerated nerves showed that large myelinated axons were present with an even distribution in both groups. In the hydrogen saline group, the total number of myelinated axons, the diameter of the regenerated axons and the degree of myelination were significantly higher than those in the normal saline group at the pre-indicated time-points after surgery $(\mathrm{P}<0.05)$ (Fig. 2), suggesting that hydrogen saline enhanced axonal regeneration and remyelination.

Hydrogen-rich saline promotes the recovery of motor function. Electrophysiological tests were performed to evaluate the effects of hydrogen saline on functional recovery. The rats in the hydrogen saline group achieved improved functional recovery, with a higher CMAP peak amplitude and NCV than those in the normal saline group at 4,8 and 12 weeks after surgery $(\mathrm{P}<0.05$, Fig. 3).
SFI is commonly used to evaluate the recovery of motor function in rat models of sciatic nerve injury. In the current study, the SFI measurements exhibited progressive increases in both groups over time, indicating motor function improvement following surgery. In the hydrogen saline group, the SFI scores were significantly higher than those in the normal saline group at the pre-indicated time-points $(\mathrm{P}<0.05)$ (Fig. 4), indicating improved recovery of motor function in rats receiving hydrogen saline compared with rats receiving normal saline during all evaluated periods.

$\mathrm{P}_{m}$ reflects the extent of gastrocnemius muscle atrophy as an index of motor function recovery. Morphometric analysis revealed that the $\mathrm{P}_{m}$ in the hydrogen saline group was significantly higher than that in the normal saline group (Fig. 5), indicating that the atrophy of the gastrocnemius muscles was partially alleviated in the hydrogen saline group.

\section{Discussion}

In the current study, the effect of hydrogen saline on axonal regeneration and functional recovery following reconstructive surgery to repair a $10-\mathrm{mm}$ nerve gap in rats was investigated. The motor function evaluation, including the SFI scores, target muscle atrophy changes and CMAP parameters, and the histomorphological observations of the regenerated nerves and FG retrograde tracing revealed that hydrogen saline improved peripheral nerve regeneration with significant functional recovery, suggesting that hydrogen-rich saline could be used for peripheral nerve injury therapy.

In contrast with the central nervous system, the peripheral nervous system has the potential to spontaneously regenerate 

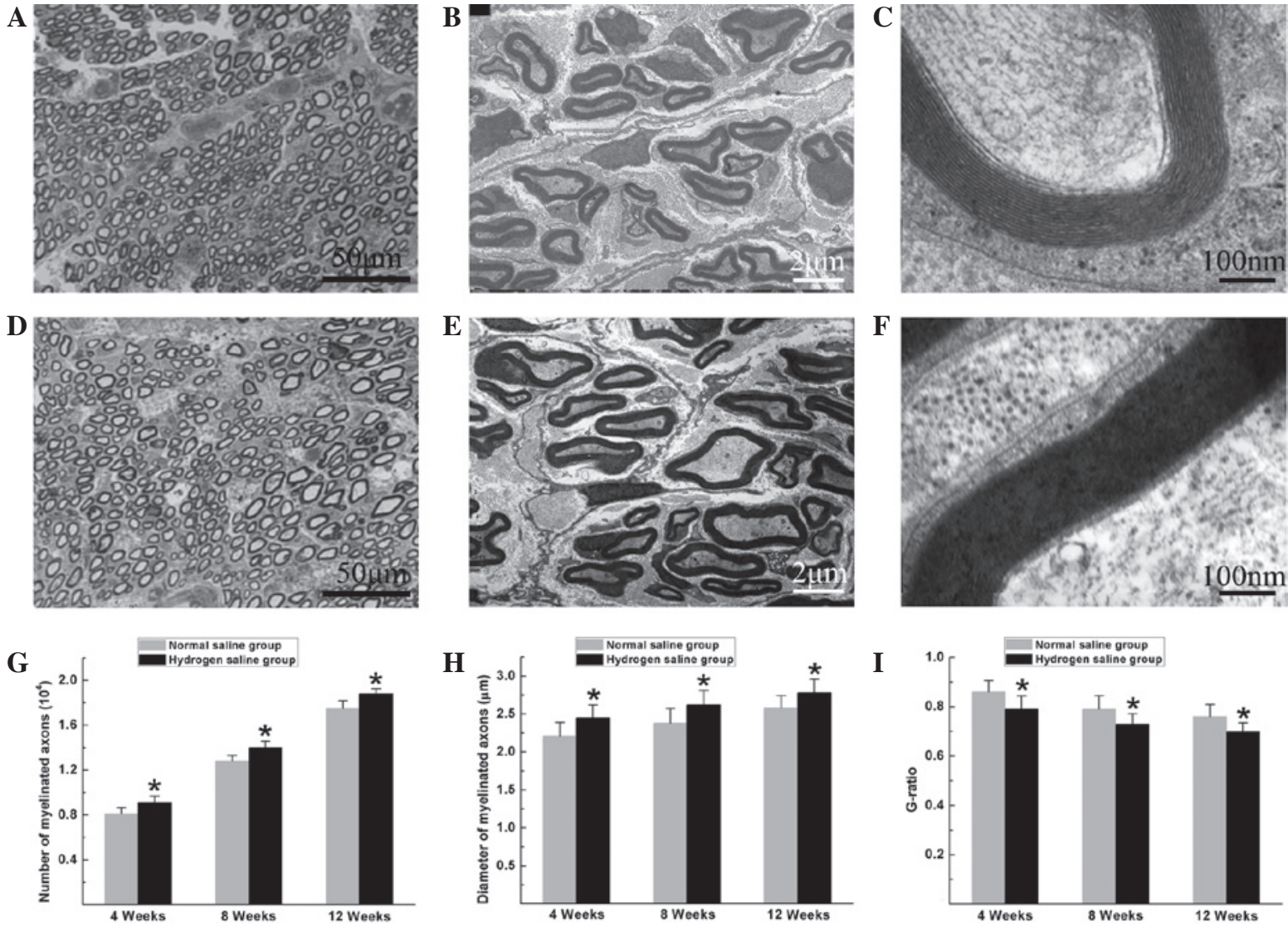

Figure 2. Histomorphological observation of the distal portion of the regenerated nerves at 12 weeks after surgery. (A-C) Normal saline group: (A) Toluidine blue staining of the regenerated axons; transmission electron micrographs of the (B) regenerated axons and (C) myelin sheath. (D-F) Hydrogen saline group: (D) Toluidine blue staining of the regenerated axons; transmission electron micrographs of the (E) regenerated axons and (F) myelin sheath. (G-I) The (G) total number of myelinated axons, (H) mean diameter of the nerve fibers and (I) G-ratio were measured. All data are expressed as the mean \pm standard error of the mean. ${ }^{*} \mathrm{P}<0.05$ for the comparison with the normal saline group.
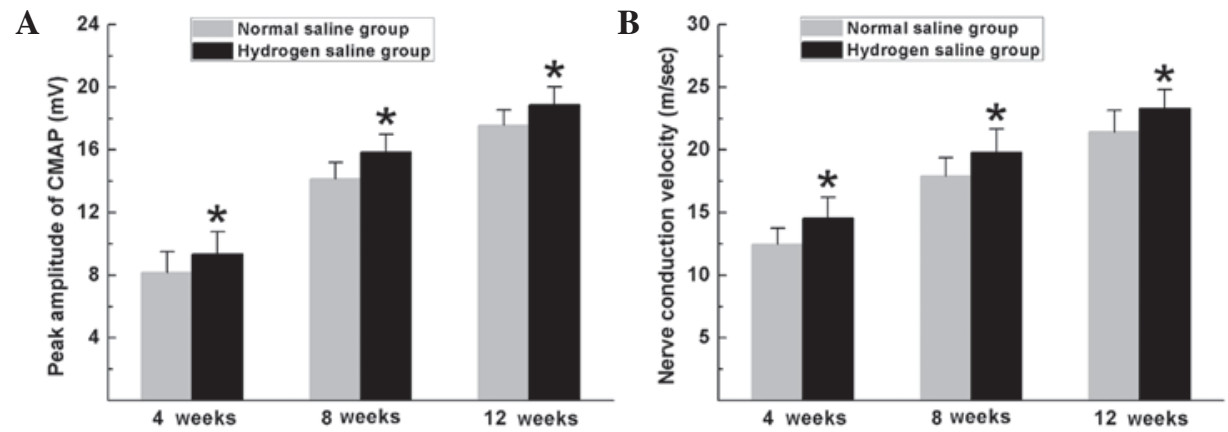

Figure 3. Electrophysiological tests were performed at 4, 8 and 12 weeks after surgery. Comparisons of (A) CMAP amplitude and (B) nerve conduction velocity. All data are expressed as the mean \pm standard error of the mean. ${ }^{*} \mathrm{P}<0.05$ for the comparison with the normal saline group. CMAP, compound muscle action potential.

following injury. Regeneration requires well-orchestrated interactions between neurons and Schwann cells. In large nerve gaps, however, regenerating axons can take considerable time to penetrate across grafts and reach distal nerve stumps. During this process, numerous apoptotic signaling pathways are activated within the axotomized neurons, resulting in a loss of neurons and limiting the efficiency of nerve repair (19-21). Oxidative stress is commonly generated following peripheral nerve injury and is reported to be a major activator of caspase activity, thereby promoting apoptosis (22-24). Thus, the use of antioxidants to alleviate oxidative stress may protect axoto- mized neurons from apoptosis and improve nerve regeneration. Hydrogen efficiently reduces the levels of ROS in vitro and exerts therapeutic antioxidant activity in vivo (7-9). Previous studies have indicated that hydrogen has the potential to exert neuroprotective effects through the suppression of oxidative stress and the reduction of neuronal apoptosis in the central nervous system (10-12). In the present study, an increased number of FG-positive neurons was observed within the spinal cord and DRG in the hydrogen saline group, suggesting that hydrogen exerted anti-apoptotic actions through the suppression of oxidative stress, thereby decreasing the number of 


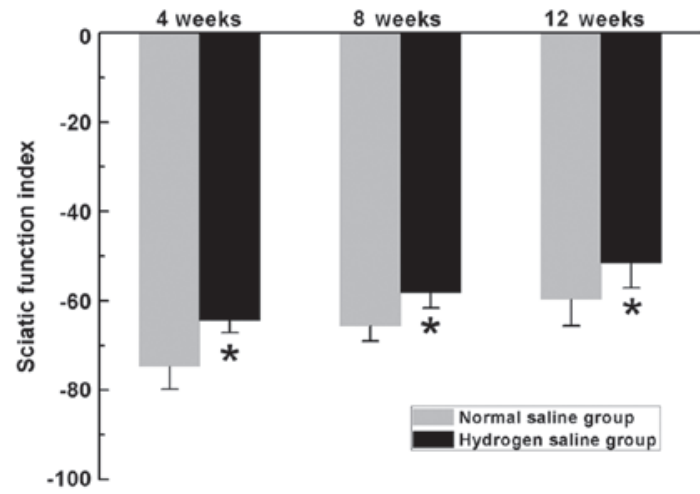

Figure 4. SFI scores in the two groups. SFI scores vary between 0 and -100 , with 0 representing normal function and -100 indicating a complete loss of motor function. All data are expressed as the mean \pm standard error of the mean. ${ }^{*} \mathrm{P}<0.05$ for the comparison with the normal saline group. SFI, sciatic function index.

apoptotic cells and maintaining more viable neurons. In addition, more nerve fibers were observed in the hydrogen saline group, suggesting that more nerve fibers were generated by the surviving neurons and regenerated into distal stumps. These observations indicated that hydrogen saline is beneficial to neuronal survival and axonal outgrowth following nerve injury; however, the exact mechanism underlying the neuroprotection of hydrogen-rich saline on peripheral nerve regeneration remains largely unknown and requires investigation in further studies.

Electrophysiological properties provide objective and reliable indices for the evaluation of nerve regeneration and motor function recovery, and histomorphological evidence of regenerated nerves provides the structural basis for electrophysiological performance. The NCV depends on the diameter of the axons and the thickness of the myelin sheath, while the amplitude of the CMAP is associated with the number of axons that achieve and reinnervate target muscles (25-27). In the present study, the larger mean diameter and thicker myelin sheath of the myelinated axons may have contributed to the increased NCV in rats receiving hydrogen-rich saline. In addition, the higher number of myelinated axons may have contributed to the higher amplitude of the CMAP, while the latter was concomitant with the improved histological appearance of gastrocnemius muscles in rats treated with hydrogen saline. These results suggest that hydrogen saline benefits axon remyelination and the reinnervation of target muscles. In peripheral nerves, the myelin sheath is formed by Schwann cells, and its thickness is regulated through neuregulin-ErbB signaling within these cells (28). It is likely that the administration of hydrogen-rich saline positively affected neuregulin-ErbB2 signaling within Schwann cells and contributed to the formation of a thicker myelin sheath in the hydrogen-rich saline group; however, additional studies are required to confirm this idea.

In combination, the results of the present study have shown the alleviation of peripheral nerve injury by hydrogen-rich saline. Axonal regeneration and functional recovery were assessed though a combination of behavioral and electrophysiological analyses, FG retrograde tracing and morphometric analyses of the regenerated nerves and target muscles. The

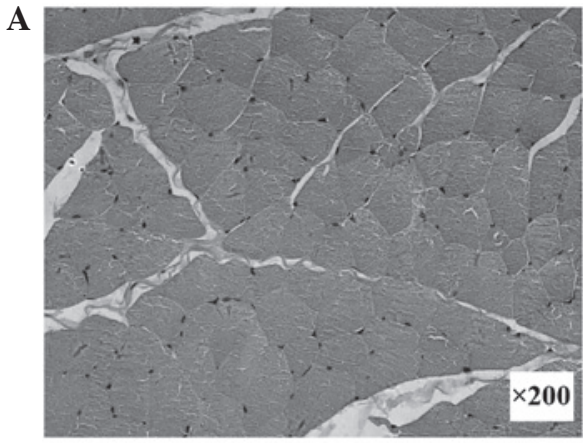

B

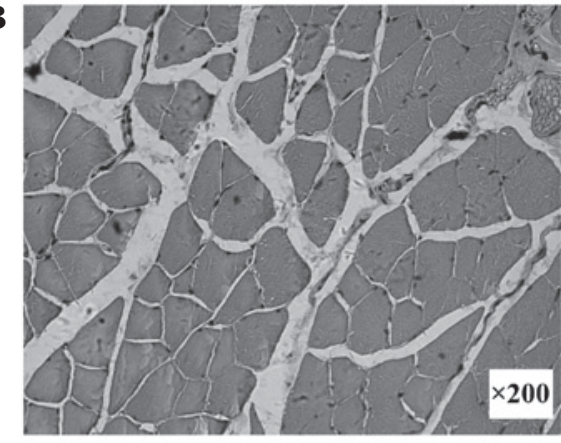

C

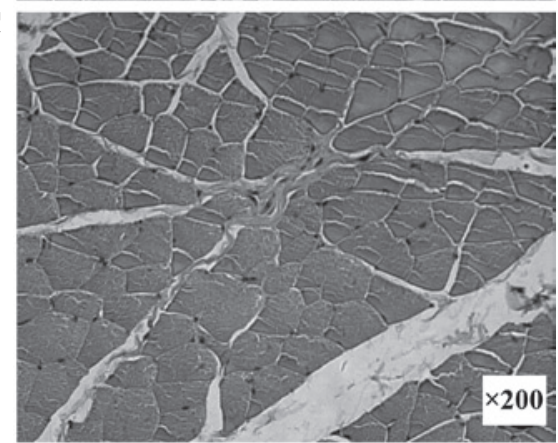

D

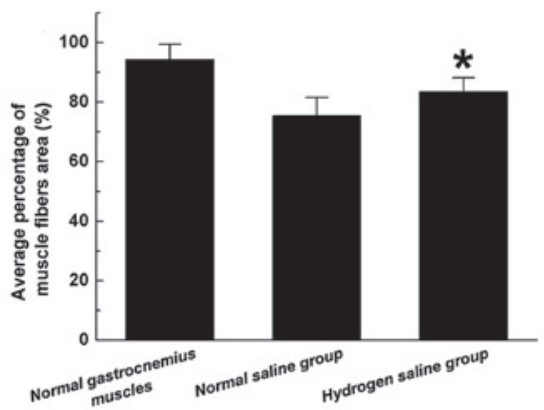

Figure 5. Light micrographs of transverse sections of the gastrocnemius muscle following hematoxylin and eosin staining at 12 weeks after surgery: (A) Contralateral control side; (B and C) operated side in the (B) normal saline and (C) hydrogen saline groups. (D) Average percentage of muscle fiber area. All data are expressed as the mean \pm standard error of the mean. ${ }^{~} \mathrm{P}<0.05$ for the comparison with the normal saline group. Magnification, $\mathrm{x} 200$.

encouraging outcomes indicated the potential application of hydrogen-rich saline for nerve regeneration therapies; however, it should be pointed out that further studies are required to investigate the beneficial effects of hydrogen-rich saline on peripheral nerve regeneration. In addition, it is unclear whether the protective effect of hydrogen-rich saline on nerve injury is dose-dependent. Furthermore, the optimal dose of hydrogen-rich saline and the duration of use for promoting nerve regeneration and functional recovery have yet to be identified. Additional studies are required to address these issues. 


\section{Acknowledgements}

This work was supported by the Nature Science Foundation of China (No. 81360408) and the Nature Science Foundation of Fujian province (No. 2015J05166). The authors would like to thank Ms. Ming-Ya Zhu and Dr Yang Li for their excellent technical assistance.

\section{References}

1. Alluin O, Wittmann C, Marqueste T, et al: Functional recovery after peripheral nerve injury and implantation of a collagen guide. Biomaterials 30: 363-373, 2009.

2. Kehoe S, Zhang XF and Boyd D: FDA approved guidance conduits and wraps for peripheral nerve injury: A review of materials and efficacy. Injury 43: 553-572, 2012.

3. Tang X, Xue C, Wang Y, Ding F, Yang Y and Gu X: Bridging peripheral nerve defects with a tissue engineered nerve graft composed of an in vitro cultured nerve equivalent and a silk fibroin-based scaffold. Biomaterials 33: 3860-3867, 2012.

4. Cui Q, Zhang J, Zhang L, Li R and Liu H: Angelica injection improves functional recovery and motoneuron maintenance with increased expression of brain derived neurotrophic factor and nerve growth factor. Curr Neurovasc Res 6: 117-123, 2009.

5. Nie X, Zhang YJ, Tian WD, et al: Improvement of peripheral nerve regeneration by a tissue-engineered nerve filled with ectomesenchymal stem cells. Int J Oral Maxillofac Surg 36: 32-38, 2007.

6. Hart AM, Terenghi G and Wiberg M: Neuronal death after peripheral nerve injury and experimental strategies for neuroprotection. Neurol Res 30: 999-1011, 2008.

7. Ohsawa I, Ishikawa M, Takahashi K, et al: Hydrogen acts as a therapeutic antioxidant by selectively reducing cytotoxic oxygen radicals. Nat Med 13: 688-694, 2007.

8. Fukuda K, Asoh S, Ishikawa M, Yamamoto Y, Ohsawa I and Ohta S: Inhalation of hydrogen gas suppresses hepatic injury caused by ischemia/reperfusion through reducing oxidative stress. Biochem Biophys Res Commun 361: 670-674, 2007.

9. Sato Y, Kajiyama S, Amano A, et al: Hydrogen-rich pure water prevents superoxide formation in brain slices of vitamin C-depleted SMP30/GNL knockout mice. Biochem Biophys Res Commun 375: 346-350, 2008

10. Cai J, Kang Z, Liu WW, et al: Hydrogen therapy reduces apoptosis in neonatal hypoxia-ischemia rat model. Neurosci Lett 441: 167-172, 2008.

11. Chen C, Chen Q, Mao Y, et al: Hydrogen-rich saline protects against spinal cord injury in rats. Neurochem Res 35: 1111-1118, 2010.

12. Yonamine R, Satoh Y, Kodama M, Araki Y and Kazama T: Coadministration of hydrogen gas as part of the carrier gas mixture suppresses neuronal apoptosis and subsequent behavioral deficits caused by neonatal exposure to sevoflurane in mice. Anesthesiology 118: 105-113, 2013.
13. Sun Q, Kang Z, Cai J, et al: Hydrogen-rich saline protects myocardium against ischemia/reperfusion injury in rats. Exp Biol Med (Maywood) 234: 1212-1219, 2009.

14. de Medinaceli L, Freed WJ and Wyatt RJ: An index of the functional condition of rat sciatic nerve based on measurements made from walking tracks. Exp Neurol 77: 634-643, 1982.

15. Hare GM, Evans PJ, Mackinnon SE, et al: Walking track analysis: A long-term assessment of peripheral nerve recovery. Plast Reconstr Surg 89: 251-258, 1992.

16. Suzuki Y, Tanihara M, Ohnishi K, Suzuki K, Endo K and Nishimura Y: Cat peripheral nerve regeneration across $50 \mathrm{~mm}$ gap repaired with a novel nerve guide composed of freeze-dried alginate gel. Neurosci Lett 259: 75-78, 1999.

17. Huang J, Lu L, Hu X, et al: Electrical stimulation accelerates motor functional recovery in the rat model of $15-\mathrm{mm}$ sciatic nerve gap bridged by scaffolds with longitudinally oriented microchannels. Neurorehabil Neural Repair 24: 736-745, 2010.

18. Novikova L, Novikov L and Kellerth JO: Persistent neuronal labeling by retrograde fluorescent tracers: A comparison between Fast Blue, Fluoro-Gold and various dextran conjugates. J Neurosci Methods 74: 9-15, 1997.

19. Fu SY and Gordon T: The cellular and molecular basis of peripheral nerve regeneration. Mol Neurobiol 14: 67-116, 1997.

20. Nicholson DW and Thornberry NA: Caspases: Killer proteases. Trends Biochem Sci 22: 299-306, 1997.

21. Vukosavic S, Dubois-Dauphin M, Romero N and Przedborski S: Bax and Bcl-2 interaction in a transgenic mouse model of familial amyotrophic lateral sclerosis. J Neurochem 73: 2460-2468, 1999.

22. Naik AK, Tandan SK, Dudhgaonkar SP, et al: Role of oxidative stress in pathophysiology of peripheral neuropathy and modulation by N-acetyl-L-cysteine in rats. Eur J Pain 10: 573-579, 2006.

23. Senoglu M, Nacitarhan V, Kurutas EB, Senoglu N, Altun I, Atli Y and Ozbag D: Intraperitoneal alpha-lipoic acid to prevent neural damage after crush injury to the rat sciatic nerve. J Brachial Plex Peripher Nerve Inj 4: 22, 2009.

24. Formichi P, Battisti C, Radi E, Di Maio G and Federico A: Apoptosis, oxidative stress and neurological disease. J Siena Acad Sci 1: 40-45, 2009.

25. Bromberg MB: Quantitative Electromyography. In: Electrodiagnosis in Clinical Neurology. Aminoff MJ (ed). 4th edition. Churchill Livingstone, New York, NY pp257-263, 1999.

26. Rosen JM, Pham HN and Hentz VR: Fascicular tubulization: A comparison of experimental nerve repair techniques in the cat. Ann Plast Surg 22: 467-478, 1989.

27. Matsumoto K, Ohnishi K, Kiyotani T, et al: Peripheral nerve regeneration across an 80 -mm gap bridged by a polyglycolic acid (PGA)-collagen tube filled with laminin-coated collagen fibers: A histological and electrophysiological evaluation of regenerated nerves. Brain Res 868: 315-328, 2000.

28. Sherman DL and Brophy PJ: Mechanisms of axon ensheathment and myelin growth. Nat Rev Neurosci 6: 683-690, 2005. 